\title{
Using Coconuts as a Model for Analysing the Injury Pattern of Cranial Blunt Trauma
}

\author{
J. Engkjaer Christensen, M.L. Jorkov and N. Lynnerup*
}

Department of Forensic Medicine, University of Copenhagen, Denmark

\begin{abstract}
Blunt trauma to the head may result in the blunt instrument leaving specific imprints or fracture patterns. Being able to correctly correlate one or more possible blunt instruments with the imprints and fractures of an actual case may be important in police investigations. However, most of our knowledge about blunt instruments and cranial imprints and fracture patterns stems from earlier tests performed on cadavers, or more recently, advanced mechanical and computerbased models. As the former is not feasible ethically, and the latter somewhat demanding in terms of laboratory settings and computer programming. Here we present our results by using a fairly simple model: a coconut shell. Striking a half coconut shell with four different blunt instruments did produce consistent imprinting and fracture patterning, which was also consistent with previously published cases. Also, in a blind trial we were able to correctly exclude one or more blunt instruments as the causative weapon. On the other hand, the blind trial also showed that the coconut is not applicable as a model to positively identify a blunt instrument unless the imprint or fracture pattern has a very characteristic shape.
\end{abstract}

\section{INTRODUCTION}

It may be critical to determine the murder weapon in cases of blunt force homicide. In some cases the police may find possible blunt instruments at the crime scene, one of which may have been the instrument used. There is then the need to be able to identify one instrument as the likely murder weapon or alternatively rule out some of the possible weapons. It has been shown that blunt force trauma is often committed with the instruments at hand, for instance tools or baseball bats $[1,2]$. It has also been stated that in most cases the blunt force trauma was inflicted on the head [2]. The skull has the ability to preserve marks left by the instrument. There are several case reports were the injury pattern in the skull has been crucial in establishing the murder weapon [3,4].

When the skull is struck with a blunt instrument there are three possible results depending on the amount of force; 1 : No mark(s) left in the skull; 2: An imprint of the instrument in the skull; 3: An impression fracture. An imprint is when there is a mark from the instrument on the outer table of the skull but no visible fracturing of the inner table of the skull. Often the contours of the imprint match the shape of the weapon. Impression fractures are fractures which often look like spider webs with fracture lines radiating outwards from the point of impact. The central part (point of impact) is pushed medially as there are fractures of both the outer and inner table of the skull. Sometimes the central fracture can show the injury pattern of the instrument [5].

In order to assist the police in their investigations, a model is needed to test and compare the patterns left by possible instruments of blunt trauma. Earlier, cadavers have been used in such cranial fracture experiments [6], but this is

*Address correspondence to this author at the Department of Forensic Medicine, University of Copenhagen, Denmark; Tel: +45 35327239; Fax: +45 35327215; E-mail: n.lynnerup@antrolab.ku.dk not ethically acceptable anymore. CT scanning with subsequent finite element analyses (FEA) is another possibility, but these analysis are still somewhat complicated and expensive [7].

Our idea was to use a coconut shell as a cheap and simple model of the skull. Half a coconut shell is roughly equivalent to the theca cranii in shape although the coconut is smaller. Furthermore, a coconut is similar to a skull in that it consists of both a hard and a soft material. The brown endocarp is a hard shell although it is not a mineralised material. The edible (white) part of the coconut (endosperm) is soft.

We conducted a series of laboratory experiments to test the ability of coconuts as a model for cranial injury patterns of blunt instruments. We first tested whether four different blunt instruments would produce different, but consistent and identifiable imprint and fracture patterns in the coconuts, and to see whether these matched the injury patterns found in verified forensic cases. The second part was a blinded study to see if we were able to correctly identify the blunt instrument causing a specific fracture pattern.

\section{MATERIALS AND METHODOLOGY}

We used four blunt instruments: a lead pipe (Fig. 1), a hammer with a round head (Fig. 2), a hammer with a square head (Fig. 3) and a crescent wrench (Fig. 4).

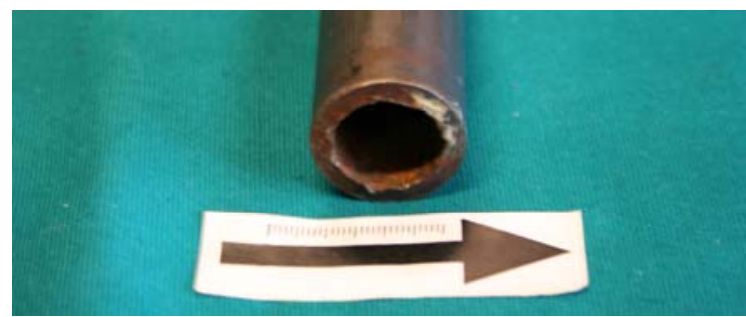

Fig. (1). Lead pipe, diameter $2.2 \mathrm{~cm}$. 


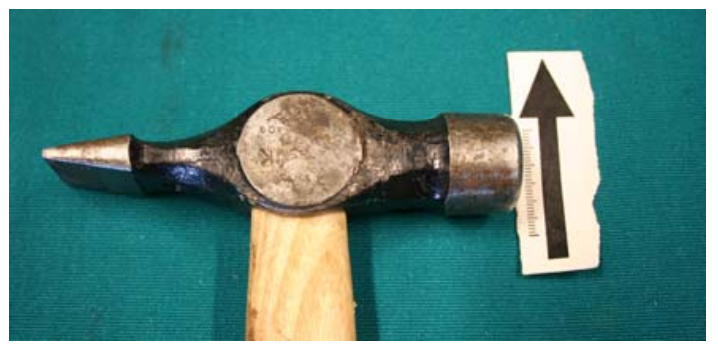

Fig. (2). Round hammer (hammerhead diameter $2.3 \mathrm{~cm}$ ).

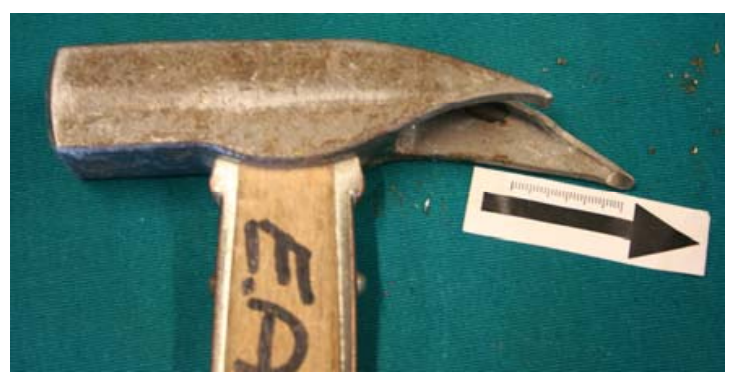

Fig. (3). Square hammer (hammerhead measures $2.8 \times 2.8 \mathrm{~cm}$ ).

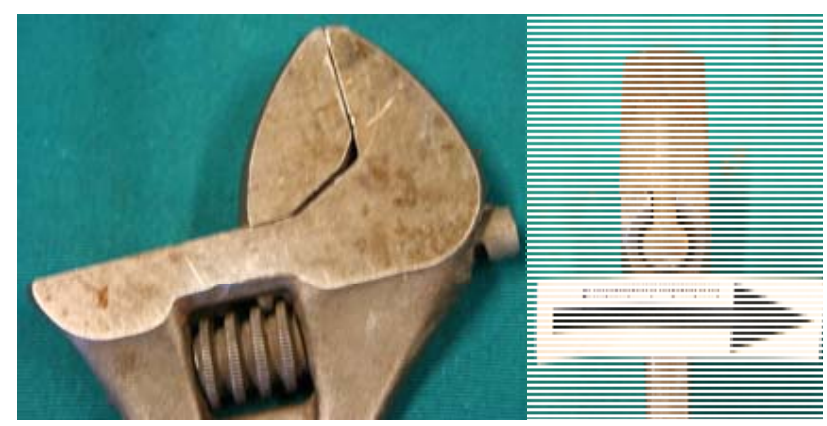

Fig. (4). Crescent wrench. The wrench is $2 \mathrm{~cm}$ thick, and has a pin with a $1.1 \mathrm{~cm}$ diameter.

The coconuts were prepared by removing the milk, after which they were sawn in half lengthwise. Each half coconut was then placed on a workbench and then hit with one of the four instruments. Through experience we found that if we hit the coconut with too little force there would be no imprint or fracturing and if we hit it with too much force it would shatter completely. If the coconut shattered it was discarded. If the instrument fractured the coconut or left an imprint in the coconut the coconut was marked with a number and the instrument used. We hit the coconuts from different angles. We assembled at least 10 half coconuts with the pattern of each instrument. We then photographed each coconut and each of the four blunt instruments using a digital SLR camera (Canon 350D).

The coconut pictures were then sorted according to which blunt instrument had been used and were then analyzed to see if the instruments left specific patterns. This was done by examining each picture individually using the ruler included in the pictures and zooming in on the fractures or the imprints left by the blunt instrument and studying these. Firstly, the coconut was checked to see if there were any missing or crushed pieces and it was checked for fractures.
Then each fracture was closely investigated to clarify if they were fracture lines or patterned fractures and to assess how many fractures of each kind there was. If they were patterned, the fractures were measured and compared to the size and shape of the instrument and it was described which part of the instrument the impression represented (edge, corner, pin etc). If there was no fracture, the coconut was examined to see if there was an imprint of the blunt instrument. Again this imprint was measured and the shape and size compared to the blunt instrument. The data were then registered for each coconut.

For the blind trial 10 coconuts were prepared as described above. The coconuts were then hit using the same four blunt instruments as used above while one of us (JEC) was not present. The coconuts were then photographed. The photographs were then examined by JEC. The coconuts were checked for patterns resembling the patterns of the four instruments found in the first part of the experiment. Then the fractures and imprints were measured and compared in shape and size to the four blunt instruments. For each case, the results of this analysis lead to one or more of the instruments being selected as possible instruments. Among the possible instruments, one was then chosen as the probable most likely instrument. The patterns were then once more compared to the four blunt instruments in order to exclude between none and three instruments. The exclusion was based on the shape and size of the patterns e.g. the square hammer could not leave a circular or semicircular injury pattern.

\section{RESULTS}

\section{Injury Patterns of the Blunt Instruments}

Hitting the coconut with the lead pipe (Fig. 1) left a fracture in nine out of ten times. It always left fracture lines and always somewhere between one and four fractures (Fig. 5). The lead pipe never left a circular patterned fracture, an imprint or an impression fracture.

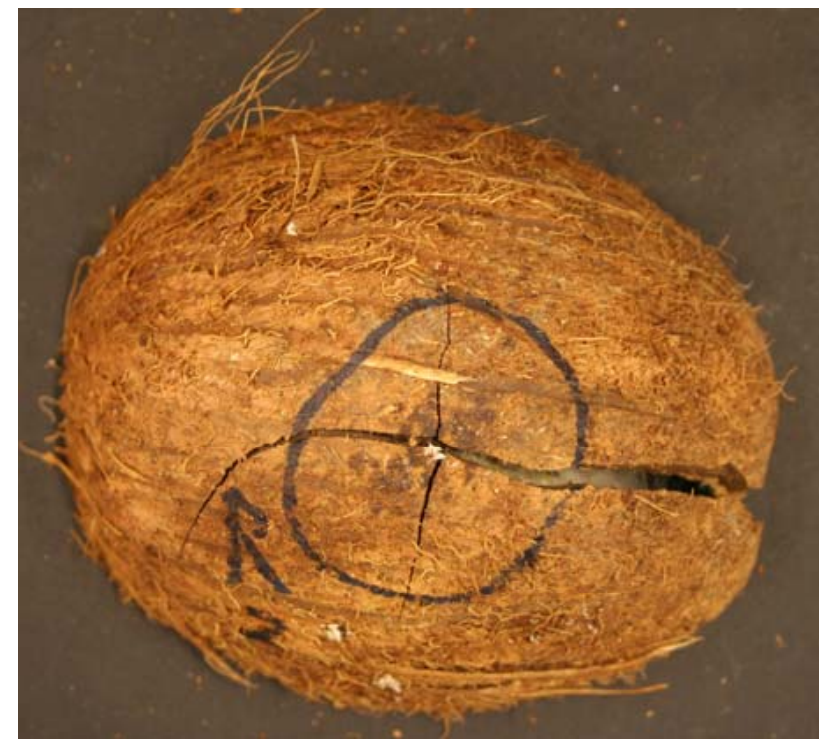

Fig. (5). Coconut struck by lead pipe (Fig. 1).

The round hammer left an impression fracture equivalent to the round hammerhead in four out of ten coconuts (Fig. 6). In two other coconuts there were imprints in the surface 
but no fractures. The imprints matched the hammer head in shape and size. All six of these impressions and prints reproduced the shape of the hammerhead, but not the full head, as they were all struck at an angle, which is most often the case in blunt force homicide cases. This means that the pattern shown is a semicircular shape which had a smaller crosssection than the largest diameter of the hammerhead.

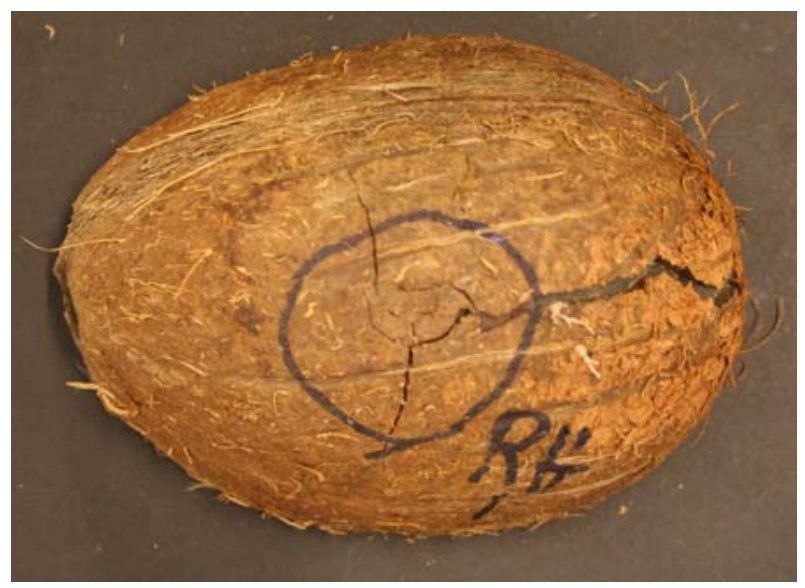

Fig. (6). Coconut struck by round hammer (Fig. 2) showing impression fracture and fracture lines.

All 12 coconuts struck by the square hammer had linear fractures (Fig. 7). Furthermore, there were six impression fractures and one imprint. All of the impression fractures and the imprint matched the size and shape of the square hammerhead. Again all of them were struck at an angle leaving a $\mathrm{V}$-shaped fracture or imprint.

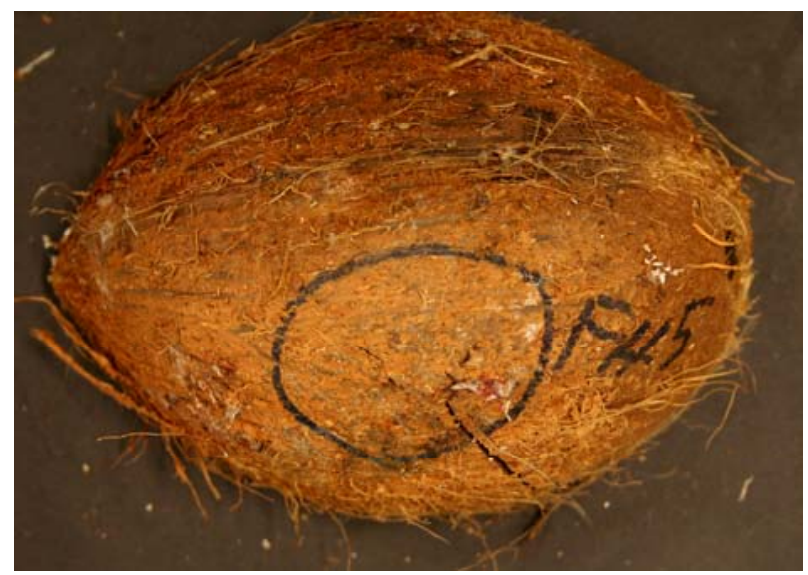

Fig. (7). Coconut struck with a square hammer (Fig. 3) showing a $\mathrm{V}$-shaped impression fracture matching the square hammer in size and shape. Furthermore a fracture line is seen.

The crescent wrench (Fig. 4) left a patterned injury in 11 out of 12 coconuts. It left impression fractures (six coconuts) or imprints (five coconuts). All of them matched the instrument in shape and size. They faithfully reproduced the shape of the pin of the crescent wrench (Fig. 8).

Overall, it can be said that the pipe left no identifying marks but always fractured the coconut. The round hammer left a defining mark in six out of ten coconuts. The square hammer fractured every coconut and in seven out of 12 coconuts it left an identifying mark. The crescent wrench left a mark of its tap in the form of a fracture or an imprint in almost all cases (see Table 1). These patterns resemble the patterns found in pictures of blunt force homicide victims $[4,8]$.

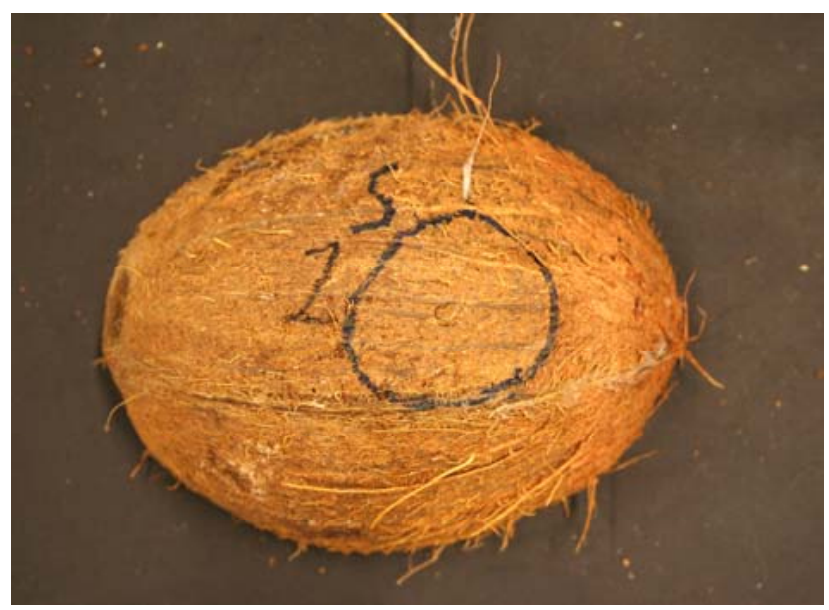

Fig. (8). Coconut struck with a crescent wrench (Fig. 4) showing an impression fracture matching the pin of the wrench in shape and size. There are no fracture lines.

Table 1. Fracture Patterns for four Blunt Instruments. The Total of Each Column can Exceed the Number of Coconuts Because a Coconut Could Have More Than One Type of Fracture

\begin{tabular}{|c|c|c|c|c|}
\hline & Pipe & $\begin{array}{c}\text { Round } \\
\text { Hammer }\end{array}$ & $\begin{array}{c}\text { Square } \\
\text { Hammer }\end{array}$ & $\begin{array}{c}\text { Crescent } \\
\text { Wrench }\end{array}$ \\
\hline \hline Fracture lines & 9 & 6 & 10 & 4 \\
\hline Impression & 0 & 4 & 6 & 6 \\
\hline Imprint & 0 & 2 & 1 & 5 \\
\hline n/a & 1 & 2 & 0 & 1 \\
\hline Number of coconuts & 10 & 10 & 12 & 12 \\
\hline
\end{tabular}

\section{Results of the Blinded Study}

It was not possible to choose any of the instruments in eight cases: all instruments could be likely instruments. In 12 cases one $(\mathrm{N}=1)$ or two $(\mathrm{N}=2)$ or three $(\mathrm{N}=9)$ instruments could be chosen (Table 2). The correctness of choosing the instruments in these cases was 9/12.

Table 2. Identification of Possible Blunt Force Instruments

\begin{tabular}{|c|c|c|}
\hline $\begin{array}{c}\text { Number of Instruments Chosen } \\
\text { as Possible Instruments }\end{array}$ & $\begin{array}{c}\text { Number of } \\
\text { Cases }\end{array}$ & $\begin{array}{c}\text { Correct } \\
\text { Assessment }\end{array}$ \\
\hline \hline All & 8 & $8(100 \%)$ \\
\hline 3 & 9 & $7(78 \%)$ \\
\hline 2 & 2 & $1(50 \%)$ \\
\hline 1 & 1 & $1(100 \%)$ \\
\hline Total & 20 & 17 \\
\hline
\end{tabular}


Having tried to choose one or more instruments as possible instruments, we then tried to choose just one weapon as the single most likely instrument. We thought we could identify one instrument as the single most likely instrument in 19/20 cases. However, this was only correct in four cases. In the cases where the instrument could not be correctly identified there seemed to be no systematic error; for instance that the square hammer was mistaken for the lead pipe or vice versa.

Table 3. Identification of Blunt Force Instruments that could not be the Instrument Used

\begin{tabular}{|c|c|c|}
\hline $\begin{array}{c}\text { Number of Instruments } \\
\text { Eliminated }\end{array}$ & $\begin{array}{c}\text { Number of } \\
\text { Cases }\end{array}$ & $\begin{array}{c}\text { Correct } \\
\text { Assessment }\end{array}$ \\
\hline \hline None & 8 & 8 \\
\hline 1 & 10 & 8 \\
\hline 2 & 1 & 0 \\
\hline 3 & 1 & 1 \\
\hline Total & 20 & 17 \\
\hline
\end{tabular}

Finally, we tested whether instead of (positively) choosing possible instruments, we might be able to exclude one or more instruments as possible instruments. Exclusion of one or more instruments was made in 12 cases. In the remaining eight cases no instruments could be ruled out (none of the instruments were in these cases excluded as possible instruments because no distinguishing mark was left on the surface of the coconut). Correct elimination of one or more instruments was performed in 9/12 cases (see Table $\mathbf{3}$ ).

\section{DISCUSSION}

It has been shown that a primary fracture starts in the area of the greatest stress [6]. From there it moves in a straight line towards and away from the area of impact. If more power is applied a secondary fracture will appear. The secondary fracture looks like the primary fracture but originates in a different area. If even more power is applied a tertiary fracture will appear at the site of impact and will expand radially. The difference in applied force between a secondary and tertiary fracture is very small hence secondary fractures are rare [6]. Overall, three forces are important in the development of fractures: the external force inflicted on the body; the internal force caused by muscle contraction or ligament tension; and the internal reaction forces between bones. Bone reacts to stress and strain depending on the stiffness, elasticity and ductility of the bone tissue and architecture [10]. The size of the fracture is determined by the velocity of the striking weapon, causing primary, secondary or tertiary fractures depending on the amount of kinetic energy deposited in the tissue. The compression increases on the outer table and tension increases on the inner table, with the opposite reactions in the areas immediately surrounding the impact site, thereby creating an internal bevelling [10].

For forensic purposes the imprint of the weapon or the fracture left by the weapon may be better evidence than markings in the skin [9]. This is because the skin only poorly preserves the shape of the weapon due to its elasticity, while the skull retains the exact pattern left in it $[3,4]$. There are several case reports were the patterned injury was crucial in establishing the murder weapon $[3,4,7]$. These imprints and fractures, especially if they are distinctive for one type of weapon, can hence be helpful in aiding the police in their investigation. They can perhaps point the police to which type of weapon they should be looking for, or alternatively used to rule out a suspected weapon, or to choose the most likely weapon out of several possible weapons. However, such observations need a practical basis, whereby possible weapons may be tested to see how imprints and fractures compare with those of the actual case. Until now, this basis has been tests carried out when ethical concerns were not a problem for using cadavers [6], and case reports with definite matching between instrument and imprints and fractures $[3,4,7]$. The need for having a test has involved the construction of advanced mechanical models, e.g. [11], to match a weapon to a crime and to reconstruct trauma. Thali et al. reported morphologically reproducible results, and their model also reproduced blunt force injuries to the skin and periost [11]. Another way of matching the weapon to the victim is the use of CT-imaging specifically cranial computed tomography (CT) [7]. The authors of this article have used superimposing of pictures of possible weapons with CT-scans performed on the victim, for instance in a case where a woman had been attacked and there were two hammers that were potential weapons [7]. The problem with both the mechanical and computer-based models is their not easy implementation, special laboratory requirements and high costs.

Our aim was to see if a coconut can be used as a very simple and low-cost model of a skull when analysing the fracture pattern left by a blunt force instrument. Due to the considerable differences between bone tissue, surrounding soft tissues and skull architecture, and the coconut shell, we knew that striking force would not be a testable variable. Our study was thus purely morphological. We expected that differences in the directions and axes of growth of the tissues might have produced very different fracture patterns between verified forensic cases and coconuts. Our study shows that on a purely morphological level, the differences are not bigger than that a coconut might be used as a model for cranial fractures. This might enable forensic scientists to easily experimentally verify a possible murder weapon or to determine if a weapon was not the likely murder weapon. While admittedly a rather down-to-earth approach, it is common for forensic scientists to seek or create models for testing the injury patterns of suspected weapons or suspects e.g. creating a dummy neck for testing hand marks in manual strangulation or testing the injury pattern of a shoe [9, 12].

The results of our experiment showed that our four blunt instruments in most cases left patterned imprint that could be used for identifying purposes. The patterns caused by the crescent wrench were similar to a blunt force homicide case, as were the patterns caused by the round hammer and the square hammer. We thus find that the similarity between the blunt force homicide cases and the patterns in the coconuts indicate that the coconut can be used as a model for testing blunt force instrument patterns. 
Taking our experiment one step further we then tried to see if we could deduce something about the instrument based on the imprints and fractures. The results of this blinded study showed that the correct blunt instrument was chosen as a possible instrument in 17 out of 20 cases although this included eight cases where all instruments were chosen. On the other hand, the correct instrument was only chosen as the single most likely one in four out of 20 coconuts. This is probably due to an interpersonal difference in striking force and impact angle when the coconuts were struck in our experiments. Perhaps the problem could be overcome by hitting more than 10 coconuts when establishing the pattern of possible instruments or by letting more than one person hit the coconuts with the possible instruments.

\section{CONCLUSION}

In this preliminary study, we found that we could produce distinct and reproducible patterns in the coconut when striking the coconut with four different instruments, and that these patterns resemble the patterns found in cases of cranial blunt force homicide victims. In blind trials we were able to correctly exclude one or more blunt instruments as the causative weapon. However, our results also show that the coconut is not applicable as a model to identify a blunt force instrument unless the imprint or fracture pattern has a very characteristic shape. This means that in a given case of blunt force to the skull, various instruments or weapons may be tried out on coconuts, and comparisons may be made between the coconut imprints and fracture patterns and those of the actual case. This may be an easy and low-cost method helpful to determine the possible or unlikely causative instruments or weapons.

\section{ACKNOWLEGDEMENTS}

Technician Soeren Lorentzen is kindly thanked use of instruments and shop.

\section{REFERENCES}

[1] Mohanty S, Mohanty MK, Panigrahi MK, Das SK. Fatal head injury in homocidal victims. Med Sci Law 2005; 45: 244-8.

[2] Murphy GK. "Beaten to death" An autopsy series of homocidal blunt force injuries. Am J Forensic Med Pathol 1991: 12; 98-101.

[3] Zugibe FT, Costello JT. Identification of a murder weapon by a peculiar blunt force injury pattern and histochemical analysis. J Forensic Sci 1985; 30: 239-42.

[4] Clark EGI, Sperry KL. Distinctive blunt force injuries caused by a crescent wrench. J Forensic Sci 1992; 37: 1172-8.

[5] Kristensen KI, Vold S. Retsmedicin - Nordisk Lærebog. In: Thomsen JL, Ed. FADL's Forlag, Copenhagen 2004; pp. 73-85 (in Danish).

[6] Gurdjian ES, Webster JE, Lissner HR. The Mechanism of Skull Fracture. Radiology 1950; 54: 313-339.

[7] Bauer M, Polzin S, Patzelt D. The use of clinical CCT images in the forensic examination of closed head injuries. J Clin Forensic Med 2004; 11: 65-70.

[8] Byers SN. Introduction to Forensic Anthropology: a Textbook, Allyn \& Bacon, Boston 2002; pp. 294-311.

[9] Rao JV. Patterned injury and its evidentiary value. J Forensic Sci 1986; 31: 768-72.

[10] Hart GO. Fracture pattern interpretation in the skull: Differentiating blunt force from ballistics trauma using concentric fracture. J Forensic Sci 2005; 50: 1276-1281.

[11] Thali MJ, Kneubuehl BP, Dirnhofer R. A "skin-skull-brain model" for the biomechanical reconstruction of blunt forces to the human head. Forensic Sci Int 2002; 125: 195-200.

[12] Kristensen ME, Lynnerup N, Sejrsen S. Comparison of handmarks in manual strangulation: An experimental study. J Forensic Sci 2006; 51: 381-385. 